\title{
Encadrer aux cycles supérieurs : Étapes, problèmes et interventions, par C. Bégin
}

Constance Denis

\section{(2) OpenEdition}

\section{Journals}

Édition électronique

URL : http://journals.openedition.org/ripes/1937

DOI : 10.4000/ripes.1937

ISSN : 2076-8427

Éditeur

Association internationale de pédagogie universitaire

Référence électronique

Constance Denis, «Encadrer aux cycles supérieurs : Étapes, problèmes et interventions, par C. Bégin », Revue internationale de pédagogie de l'enseignement supérieur [En ligne], 35(1) | 2019, mis en ligne le 17 mai 2019, consulté le 25 septembre 2020. URL : http://journals.openedition.org/ripes/1937 ; DOI : https://doi.org/10.4000/ripes.1937

Ce document a été généré automatiquement le 25 septembre 2020.

Article L.111-1 du Code de la propriété intellectuelle. 


\title{
Encadrer aux cycles supérieurs : Étapes, problèmes et interventions, par C. Bégin
}

\author{
Constance Denis
}

1 Qu'est-ce que l'encadrement aux cycles supérieurs? Comment s'y préparer? Comment puis-je aider les étudiants des cycles supérieurs à conduire, de manière autonome, une recherche apportant une contribution originale à leur discipline? Bégin (2018) se propose de répondre à ces questions dans son livre Encadrer aux cycles supérieurs. Étapes, problèmes et interventions paru aux Presses de l'Université du Québec. Avec cet ouvrage, l'auteur s'adresse aux intervenants qui s'interrogent sur l'encadrement.

2 Bégin (2018) discute d'encadrement aux cycles supérieurs notamment en décrivant des situations personnelles ou provenant de rencontres et de témoignages. Divisé en 15 chapitres, le livre permet de dresser le portrait des conditions étudiantes, des étapes préparatoires à l'encadrement, les étapes de celui-ci ainsi que les problèmes et les situations critiques. Dès les premières lignes, l'auteur prend soin de proposer un vocabulaire commun tiré, entre autres, des rapports de l'Association des doyens des études supérieures au Québec et facilitant, à notre avis, la compréhension commune de l'encadrement aux cycles supérieurs. À défaut de présenter une définition concise et claire de l'encadrement, Bégin (2018) stipule qu'il s'agit d'un accompagnement dans un processus de formation à la recherche.

Dans les deux premiers chapitres, l'auteur présente un portrait de la persévérance scolaire aux cycles supérieurs. Il y aborde, entre autres, certaines conditions gagnantes comme les habiletés en lecture dans le but d'analyser les écrits, de les commenter et de les critiquer afin de les mettre au service de nouvelles contributions. Certains tableaux proposent d'ailleurs un portrait des principaux facteurs associés à la diplomation. Le lecteur pressé pourra facilement repérer les sections susceptibles de l'intéresser à l'aide de ces repères visuels.

4 Dans la deuxième partie de l'ouvrage, Bégin (2018) poursuit avec deux chapitres où il évoque le recrutement de l'étudiant en présentant trois outils concrets dont une liste 
de questions pour la première rencontre, sans compter celles qui parsèment le chapitre. À ce titre, le troisième chapitre outille habillement les nouveaux directeurs de recherche ou ceux qui cherchent à se « réinventer ». Dans le quatrième chapitre, Bégin (2018) recommande quatre règles d'or pour faciliter les modalités de rencontre et de suivi et rendre le tout agréable et efficace.

5 La section suivante décrit la fluctuation de l'accompagnement dans les différentes étapes du parcours. À ce propos, l'annexe 5.1, présentée dans le cinquième chapitre, nous apparaît comme un guide réflexif avec un fort potentiel. Quatre styles d'encadrement sont présentés, tout en guidant le lecteur parmi les limites de l'accompagnement. Les chapitres suivants glissent sur papier plusieurs principes pour faciliter le processus rédactionnel - recension des écrits, sélection de la problématique, rédaction de la question de recherche, etc. - et, en ce sens, se veulent didactiques. Bégin (2018) prend le soin de détailler des situations générales afin d'aider le lecteur à entamer une réflexion et à explorer, par lui-même, des solutions avec les pistes d'intervention mentionnées. Certaines sections se veulent plus rassurantes ou réconfortantes que d'autres, dont un encadré à la page 326 où l'auteur présente le nombre de commentaires d'une version à l'autre. Bégin (2018) poursuit d'ailleurs en discutant de la rétroaction qui va au-delà de la simple correction dans les travaux des étudiants.

6 L'ouvrage se conclut sur une partie qui traite des éléments qui affectent la progression de l'étudiant et la qualité de la relation d'encadrement. Ces chapitres, fort intéressants, présentent un - trop? - grand nombre de situations personnelles. Bégin (2018) propose des pistes d'intervention sans nécessaire stimuler une pratique réflexive. Le nouveau directeur à la lecture de ces situations pourrait se sentir seul face aux défis qui s'annoncent.

7 Malgré les situations éclairantes et les propos fort intéressants, le manque de références aux écrits scientifiques a tendance à diminuer le caractère rigoureux de l'ouvrage. La lecture est agréable, mais la posture de l'auteur se veut parfois " paternelle ». Il aurait été, à notre avis, intéressant d'ajouter des pistes de réflexion et d'utiliser les outils communicationnels pour créer une communauté de lecteurs. En effet, comme le souligne lui-même l'auteur, l'encadrement se développe par l'expérience qu'on en fait soi-même. Le livre se veut donc un outil efficace pour « contrer » cet apprentissage expérientiel. Les situations décrites, parfois personnelles ou particulières, ne reflètent peut-être pas ce que l'auteur annonce en conclusion comme étant une démarche en constante évolution selon les intervenants, les conditions, les événements, etc. Le caractère dual, faire ou ne pas faire, de certaines définitions ou interventions peut empêcher d'entrevoir toute la complexité de l'encadrement aux cycles supérieurs. Néanmoins, l'ouvrage s'inscrit dans un espace où la littérature francophone se fait rare. Il présente une lecture rapide - aucune nécessité de lire un chapitre après l'autre - et agréable sans trop de technicité. 


\section{BIBLIOGRAPHIE}

Bégin, C. (2018). Encadrer aux cycles supérieurs: Étapes, problèmes et interventions. Québec, Canada : Presses de l'Université du Québec

\section{AUTEUR}

\section{CONSTANCE DENIS}

Université de Sherbrooke, Canada

constance.denis@usherbrooke.ca 